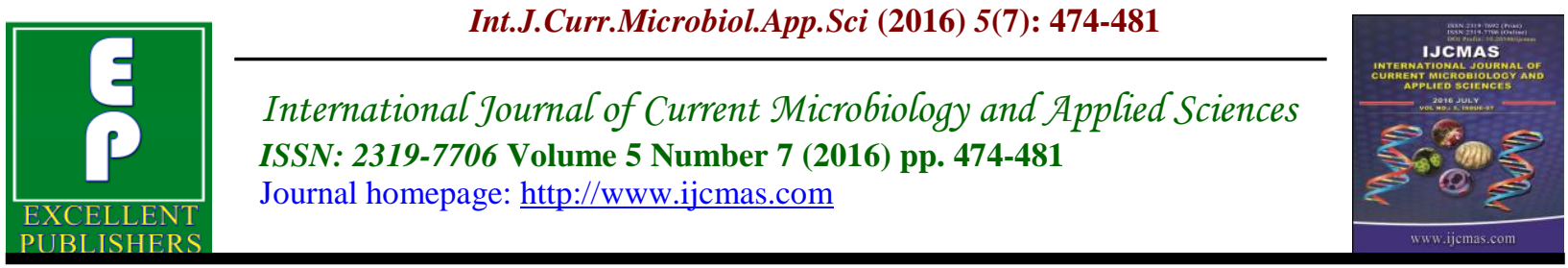

Review Article

http://dx.doi.org/10.20546/ijcmas.2016.507.051

\title{
Mitochondrial Biogenesis in Heart: Way of Nurturing and Failure
}

\author{
Swati Sharma* and Abha Swarup \\ Department of Zoology and Applied Aquaculture, Barkatullah University, Bhopal, India \\ *Corresponding author
}

\begin{abstract}
A B S T R A C T
\section{Keywords}

Mitochondria, Neurodegenerative diseases, OXPHOS, Biogenesis, Heart failure.

\begin{tabular}{l}
\hline Article Info \\
\hline Accepted: \\
15 June 2016 \\
Available Online: \\
10 July 2016
\end{tabular}

Mitochondria is the key organelle of cell which provide energy output for various functions and mechanisms inside the cell with the production of ATP and it is directly linked to the protection from chronic diseases such as neurodegenerative diseases, heart disease, diabetes and metabolic syndrome. Its biogenesis get affected by the alterations in both nuclear and mitochondrial DNA (mtDNA) due to the improper oxidative phosphorylation process. One possible approach to relieve this deficiency might be to boost the residual OXPHOS capacity by increasing the mitochondrial functional mass in the affected tissues. Heart is the organ which continuously contract and pump blood to whole body and this contraction is maintained by energy output from mitochondria. So the biogenesis and mechanisms of mitochondria is directly proportional to the working capacity and quality of heart health. Current treatments for heart disease address the symptoms of deficiency but not the cure. One possible approach to relieve the deficiency might be to boost the residual OXPHOS capacity by increasing the mitochondrial functional mass in the affected tissues which leads to the proper biogenesis of mitochondria and heart function.
\end{abstract}

\section{Introduction}

Mitochondria are double membrane subcellular organelles, and also known as the power house of the cells which is responsible for producing the major portion of cellular adenosine triphosphate (ATP) by oxidative phosphorylation (OXPHOS) (van der et al., 2005). Mitochondria is composed of two membrane structure the inner membrane and the outer membrane and the in between space is known as intermembrane space. Outer membrane has many protein based pores which allows passage of ions and molecules as well as small proteins. Inner membrane is less permeable and loaded with proteins which involved in electron transport and ATP synthesis. The electron Transport chain (ETC) consist of about 80 different polypeptides, which are organized into five transmembrane protein complexes (I-V). These protein complexes push protons to intermembrane space which creates concentration gradient of protons. The Proton gradient generated by complex I, III and IV is released through ATP synthase or complex $\mathrm{V}$, which results in phosphorylation and adenosine diphosphate to adenosine triphosphate (ATP) (Okuno et al., 2011). 
In addition to the OXPHOS machinery, mitochondria are also known as metabolic signaling center of all the cells (Nunnari et al., 2012) performing many important biological functions such as regulation of apoptosis (Kujoth et al., 2005; Iwasawa et al., 2011) maintenance of cytosolic calcium homeostasis, lipid biosynthesis (Voelker, 2009; De et al., 2011) and iron Sulphur cluster biogenesis (vander et al., 2005). Mitochondrial oxidative phosphorylation contribute the primary energy (ATP) supply in the myocardial cell. Although heart mitochondria are capable of rapidly accumulating significant amounts of calcium, their role in myocardial calcium metabolism is probably like a "cytosolic buffer or reservoir" system. Alterations due to oxidative stress in mitochondrial functions leads to cardiac disease. An increasing number of studies found mitochondrial dysfunction as the root of all disease, most notably in the heart and the central nervous system. The calcium accumulation into mitochondria may play a key role as a trigger to mitochondrial degeneration, especially when that calcium uptake is accompanied by another stressor, in particular nitrosative or oxidative stress. Oxidative stress is an imbalance between the systemic manifestation of reactive oxygen species and a biological system's ability to readily detoxify the reactive intermediates or to repair the resulting damage during lipid peroxidation. There are two major respiratory chain regions where ROS are produced, one being complex I (NADH coenzyme Q reductase) and the other complex III (ubiquinol cytochrome c reductase). The major process involved is the opening of the mitochondrial permeability transition pore, a large conductance pore which causes a collapse of the mitochondrial membrane potential and leads to ATP depletion and necrotic cell death or to cytochrome $\mathrm{c}$ release and apoptosis, depending on the rate of ATP consumption. These are the major source of reactive oxygen species (ROS), which can influence homeostatic signaling pathways, controlling the cell differentiation and proliferation (Hamanaka et al., 2010).

Mitochondrial biogenesis is the key process by which cells increase their individual mitochondrial mass and copy number. Mitochondria are produced from the transcription and translation of genes both in the nuclear genome and in the mitochondrial genome. Mitochondrial biogenesis results in more mitochondrial tissues which increases metabolic enzymes for glycolysis, oxidative phosphorylation and ultimately a greater mitochondrial metabolic capacity in cells. Mitochondrial degeneration leads to various dysfunctions in cells, tissues and organs.

Mitochondrial dysfunctions are associated with a large proportion of human diseases, such as neurodegenerative disorders, cardiovascular disorders, neurometabolic diseases, cancer, obesity, etc. (Greaves et al., 2012; McFarland et al., 2009). These groups of diseases showing tissue-specific manifestations and affecting multiple organ systems. During the last two decades, there has been a rise in the studies related to the degeneration of mitochondrial DNA (mtDNA) mutations, and their role in the expression of disease phenotype. Although earlier considered to be a rare class of disorders, with about one in one million people being affected, the So, we can say mitochondria is heart of all the functions and its degeneration leads to major damage to the cell. At present, there is no definite cure for mitochondrial disorders, with most of the available treatments being directed towards relieving the symptoms (Suomalainen, 2011). Proper diagnosis of mitochondrial disorders is very important. As mechanisms of mitochondrial disorders are not fully 
understood, they remain the major challenge with respect to its diagnosis and treatment. In this review, we present an overview of mitochondrial biogenesis in heart function and failure.

\section{Energy Output Mechanism in Mitochondria}

Mitochondrial biogenesis is the result of processes and events from the growth and division of pre-existing organelles is mitochondrial biogenesis. According to the endosymbiotic theory, mitochondria are the descendants of a proteobacteria that became established in a host cell by the process of endosymbiosis. Due to their bacterial origin, mitochondria having their own genome and the capacity for auto replication. Mitochondrial proteins are encoded by both the genome mitochondrial genome as well as the nuclear genome. Thus, this requires a special coordination between protein synthesis, nuclear encoded protein import and assembly with mitochondrial encoded proteins. This process has to occur harmoniously with the synthesis of phospholipids and mitochondrial membranes. Furthermore, mitochondrial biogenesis is dependents upon the mitochondria's ability to undergo fission/fusion which ensures proper organization of the mitochondrial network.

In vertebrates, the circular mitochondrial DNA (mtDNA) is $\sim 16.5 \mathrm{~kb}$, doublestranded, contains 37 genes encoding 13 subunits of respiratory complexes I, III, IV and $\mathrm{V}, 2$ ribosomal RNAs and 22 transfer RNAs necessary for the translation. Mitochondrial DNA is a multicopy genome and somatic mammalian cells generally have 103 - 104 copies of it, with 2 10 genomes per organelle. Other mitochondrial proteins encoded by the nuclear genome include remaining subunits of the oxidative phosphorylation system (OXPHOS), enzymes and proteins involved in pyruvate oxidation, tricarboxylic acid cycle (TCA), fatty acid oxidation (FAO), stress responses and transport. Recent integrative proteomic and biochemical data estimated that some 1,500 different proteins, corresponding to 1,080 genes, are found in mitochondria (Calvo et al., 2006).

To ensure proper mitochondrial function, nuclear-encoded proteins have to be correctly targeted and imported from the nucleus to the mitochondria (Baker et al., 2007; Hood et al., 2004). Nuclear mRNAs are translated in the cytosol to precursor proteins with mitochondria-targeting sequences. They are then escorted and unfolded by molecular chaperones and finally imported into the mitochondrial matrix via the translocase of the outer membrane (TOM) and the translocase of the inner membrane (TIM) in a membrane potential-dependent manner. Subsequently, intramitochondrial proteins cleave the targeting sequences and refold the proteins into their mature conformation. Subunits of the respiratory chain complexes are assembled with mitochondrial encoded subunits and inserted in the inner membrane by mitochondrial export machinery (Hood et al., 2004). The transcriptional regulatory mechanisms controlling mitochondrial biogenesis are essential for the co-ordination of mitochondrial and nuclear genome expression (Finck et al., 2007; Puigserver et al., 2003; Scarpulla, 2008).

Replication, maintenance and transcription of mitochondrial DNA are driven by the nuclear encoded mitochondrial transcription factor A (mtTFA) and two transcription specific factors (TFB1M and TFB2M), a single RNA polymerase (POLRMT) and a termination factor (mTERF) (Scarpulla RC, 2008). By binding an upstream enhancer of 
the two mitochondrial DNA strands, mtTFA promotes mitochondrial encoded gene transcription.

The coordination between mitochondrial and nuclear gene expression is controlled by the interplay between specific transcription factors (i.e., the nuclear respiratory factors (NRFs), the peroxisome-proliferator activated receptors (PPARs), the estrogen related receptors (ERR) and others) and members of the transcriptional co-activator of peroxisome proliferator activated receptor (PPAR) gamma (PGC-1) family. NRF1 and/or NRF2 not only controls the expression of mtTFA expression is under the control of NRF1 and/or NRF2. Moreover, NRFsbut are responsible for transcriptional activation of many nuclear-encoded mitochondrial proteins (Kelly et al., 2004), including the vast majority of genes required for the five respiratory complexes. ERRs conducts a comprehensive cardiac transcriptional program comprising uptake of energy substrates, production and transport of ATP across the mitochondrial membranes, and intracellular fuel sensing (Dufour et al., 2007). The transcription factor PPAR (peroxisome proliferator activated receptor alpha) regulates the FAO enzymes and fatty acid transporter expression is not regulated by NRFs, but instead, by the transcription factor PPAR (peroxisome proliferator activated receptor alpha) (Huss et al., 2004).

The upstream regulator of the transcriptional pathway controlling mitochondrial biogenesis is the co-activator, PGC-1. PGC1 lacks DNA-binding activity; it interacts with and co-activates a number of transcription factors, including NRFs. Mitochondrial biogenesis and respiration are stimulated by PGC-1 through powerful induction of NRF1 and NRF2 gene expression. PGC-1 increases NRFs transcriptional activity on the promoter of mtTFA (Wu et al., 1999). Moreover, PGC-1 interacts with and co-activates PPAR and its partner, the retinoid $\mathrm{X}$ receptor (RXR) (Finck, 2007; Vega et al., 2000). PGC-1 is also involved in FAO and nuclear-encoded OXPHOS gene expression and acts by coactivating the estrogen-related receptor (ERR) (Scarpulla, 2008) PGC-1 is enriched in tissues with high oxidative activity such as heart and brown adipose tissue, and it is rapidly induced under conditions of increased energy demand such as cold, exercise, and fasting. PGC-1 levels correlate with mitochondrial protein levels, mitochondrial mass and cardiac oxidative capacity, suggesting that PGC-1 is a master regulator of mitochondrial biogenesis and energy metabolism (Garnier et al., 2003; Lehman et al., 2000). PGC-1 -deficient mice exhibit a cardiac dysfunction associated with a lessened ability to in-crease work in response to physiological stimuli. However, hearts lacking PGC-1 have a normal mitochondrial volume density, although, mitochondrial gene expression, oxidative capacity and fatty acid oxidation are reduced. (Lehman et al., 2008; Leone et al., 2005).

PGC-1 was the first-discovered member of a family of three related proteins that control major metabolic functions. Whereas the PGC-1-related coactivator (PRC) is expressed ubiquitously, PGC-1 and are enriched in mitochondria rich tissues. Deficiency in PGC-1 in the heart results in a general defect in the expression of genes encoding components of the electron transport chain and also a reduced mitochondrial volume fraction, leading to a blunted response to dobutamine stimulation (Lelliott et al., 2006). In addition to the regulated expression by various metabolic stimuli, the family of PGC-1 co-activators is also controlled by post- translational 
modifications including phosphorylation, deacetylation and methylation (Ventura et al., 2008). PGC-1 is a critical regulatory molecule involved in the control of cardiac mitochondrial number and its functions.

\section{Mitochondrial Degeneration in Heart Failure}

Mitochondria and oxidative metabolism plays key role in the cardiac work and the body's needs. Continuous contractile activity of the myocardium requires regular energy production, which is $95 \%$ supplied by mitochondrial respiration, mostly from fatty acid metabolism. The central role of mitochondria in cardiac contractility involves specific regulations and adaptations of mitochondrial network structure and function. This results from mitochondrial dynamic and biogenesis, which are responsible for mitochondrial growth, organization and mitochondrial component synthesis. Heart failure is a complex syndrome associated with a significant mortality. The mechanisms involved in this represents a major challenge. Energy metabolism and mitochondrial biogenesis disorders appear to play an important role in cardiac dysfunction and progression to heart failure.

When mechanisms are exceeded, or, when the pathological stimulus occurs rapidly, the unction of heart deteriorates and shifts towards heart failure. It is a chronic syndrome in which the heart is not able to satisfy the metabolic and functional needs of the body.Regarding energy metabolism and mitochondrial biogenesis studies, there seems to be a consensus. Mitochondrial function is dramatically altered either in failing hearts of the dog (Marin et al., 2001; Sharov et al., 1998) rodent (De et al., 1999; Garnier et al., 2003; Javadov et al., 2005; Jullig et al., 2008) and human (Mettauer et al., 2006; Sharov et al., 2000).
This dysfunction occurs by a downregulation of genes controlling mitochondrial biogenesis. Decreased cardiac PGC-1 expression was first reported in an experimental model of heart failure induced by thoracic aortic constriction (Garnier et al., 2003) and subsequently in numerous models of heart failure, such as experimental myocardial infarction (Kemi et al., 2007; Sun et al., 2007) aortic constriction (Witt et al., 2008) and in human dilated cardiomyopathy and ischemic disease (Sebastiani et al., 2007). Moreover, downregulation of NRFs and mtTFA and their downstream targets (citrate synthase and cytochrome c oxidase) was also reported (Garnier et al., 2003; Javadov et al., 2005; Jullig et al., 2008; Kemi et al., 2007; Sebastiani et al., 2007). Down-regulation of the whole mitochondrial transcription cascade occurs during heart failure. Interestingly, a sex-dependent difference was observed in mice after TAC, with thirty five genes controlling mitochondrial proteins, including PGC-1, being more affected in males than females (Witt et al., 2008). In parallel to altered mitochondrial biogenesis, the failing heart also exhibits a decrease in PPAR content, correlated with FAO gene down-regulation and reduced fatty acid utilization (Sack et al., 1998; Sebastiani et al., 2007). These alterations in fatty acid metabolism seem to occur in advanced or end-stage heart failure, but not in early stages of the disease where normal rates of fatty acid oxidation were measured by (Chandler et al., 2004).

In conclusion, these findings suggest that the failing of heart can't control or maintain normal mitochondrial Biogenesis or functions because of down regulation of transcriptional pathway which controls whole process of mitochondrial biogenesis. One more interesting study was found in which Sex dependent difference was also observed during the process in which males 
are more affected than the females in mice. So the biogenesis of mitochondria in heart is the base of healthy heart function. Therefore, we can conclude that the series of these events in mitochondria correlate with cardiac dysfunction and subsequently failing of heart in the body.

\section{References}

Baker, M.J., Frazier, A.E., Gulbis, J.M., Ryan, M.T. 2007. Mitochondrial protein-import machinery: correlating structure with function. Trends Cell Biol., 17: 456-464.

Chinnery, P.F., Johnson, M.A., Wardell, T.M., Singh-Kler, R., Hayes, C., Brown, D.T., et al. 2000. The epidemiology of pathogenic mitochondrial DNA mutations. Ann. Neurol., 48: 188-93.

Chandler, M.P., Kerner, J., Huang, H., Vazquez, E., Reszko, A., Martini, W.Z., Hoppel, C.L. et al. 2004. Moderate severity heart failure does not involve a downregulation of myocardial fatty acid oxidation. Am. J. Physiol. Heart Circ. Physiol., 287, H1538-H1543.

Calvo, S., Jain, M., Xie, X., Sheth, S.A., Chang, B., Goldberger, O.A., Spinazzola, A. et al. 2006. Systematic identification of human mitochondrial disease genes through integrative genomics. Nat. Genet., 38, 576-582.

De Sousa, E., Veksler, V., Minajeva, A., Kaasik, A., Mateo, P., Mayoux, E., Hoerter, J. et al. 1999. Subcellular creatine kinase alterations. Implications in heart failure. Circ. Res., 85: 68-76.

Dufour, C.R., Wilson, B.J., Huss, J.M., Kelly, D.P., Alaynick, W.A., Downes, M., Evans, R.M. et al. 2007. Genomewide orchestration of cardiac functions by the orphan nuclear receptors ERR alpha and gamma. Cell Metab, 5, 345-
356.

De Stefani, D., Raffaello, A., Teardo, E., Szabo, I., Rizzuto, R. 2011. A forty kilo Dalton protein of the inner membrane is the mitochondrial calcium uniporter. Nature, 476: 33640.

Elliott, H.R., Samuels, D.C., Eden, J.A., Relton, C.L., Chinnery, P.F. 2008. Pathogenic mitochondrial DNA mutations are common in the general population. Am. J. Hum. Genet., 83: 254-60.

Finck, B.N. 2007. The PPAR regulatory system in cardiac physiology and disease. Cardiovasc. Res., 73, 269277.

Finck, B.N., Kelly, D.P. 2007. Peroxisome proliferator-activated receptor gamma coactivator-1 (PGC-1) regulatory cascade in cardiac physiology and disease. Circulation, 115, 2540-2548.

Garnier, A., Fortin, D., Delomenie, C., Momken, I., Veksler, V., VenturaClapier, R. 2003. Depressed mitochondrial transcription factors and oxidative capacity in rat failing cardiac and skeletal muscles. J. Physiol., 551, 491-501.

Greaves, L.C., Reeve, A.K., Taylor, R.W., Turnbull, D.M. 2012. Mitochondrial DNA and disease. J. Pathol., 226: 274-86.

Hood, D.A., Joseph, A.M. 2004. Mitochondrial assembly: protein import. Proc. Nutr. Soc., 63, 293-300.

Huss, J.M., Kelly, D.P. 2004. Nuclear receptor signaling and cardiac energetics. Circ. Res., 95, 568-578.

Hamanaka, R.B., Chandel, N.S. 2010. Mitochondrial reactive oxygen species regulate cellular signaling and dictate biological outcomes. Trends Biochem. Sci., 35: 505-13.

Iwasawa, R., Mahul-Mellier, A.L., Datler, C., Pazarentzos, E., Grimm, S. 2011. 
Fis 1 and Bap31 bridge the mitochondria-ER interface to establish a platform for apoptosis induction. EMBO, 30: 556-68.

Javadov, S., Huang, C., Kirshenbaum, L., Karmazyn, M. 2005. NHE-1 inhibition improves impaired mitochondrial permeability transition and respiratory function during postinfarction remodeling in the rat. J. Mol. Cell Cardiol., 38, 135-143.

Jullig, M., Hickey, A.J., Chai, C.C., Skea, G.L., Middleditch, M.J., Costa, S., Choong, S.Y. et al. 2008. Is the failing heart out of fuel or a worn engine running rich? A study of mitochondria in old spontaneously hypertensive rats. Proteomics, 8, 2556-2572.

Kelly, D.P., Scarpulla, R.C. 2004. Transcriptional regulatory circuits controlling mitochondrial biogenesis and function. Genes Dev, 18, 357-368.

Kujoth, G.C., Hiona, A., Pugh, T.D., Someya, S., Panzer, K., Wohlgemuth, S.E., et al. 2005. Mitochondrial DNA mutations, oxidative stress, and apoptosis in mammalian aging. Sci., 309: 481-4.

Kemi, O.J., Hoydal, M.A., Haram, P.M., Garnier, A., Fortin, D., VenturaClapier, R., Ellingsen, O. 2007. Exercise training restores aerobic capacity and energy transfer systems in heart failure treated with losartan. Cardiovasc Res, 76, 91-99.

Kisler, J.E., Whittaker, R.G., McFarland, R. 2010. Mitochondrial diseases in childhood: a clinical approach to investigation and management. Dev. Med. Child Neurol., 52: 422-33.

Lehman, J.J., Barger, P.M., Kovacs, A, Saffitz, J.E., Medeiros, D.M., Kelly, D.P. 2000. Peroxisome proliferatoractivated receptor gamma coactivator1 promotes cardiac mitochondrial biogenesis. J. Clin. Invest., 106, 847-
856.

Leone, T.C., Lehman, J.J., Finck, B.N., Schaeffer, P.J,. Wende, A.R,. Boudina, S., Courtois, M. et al. 2005. PGC1alpha deficiency causes multi-system energy metabolic derangements: muscle dysfunction, abnormal weight control and hepatic steatosis. PLoS Biol., 3, e101.

Lelliott, C.J., Medina-Gomez, G., Petrovic, N., Kis, A., Feldmann, H.M., Bjursell, M., Parker, N. et al. 2006. Ablation of PGC-1beta results in defective mitochondrial activity, thermogenesis, hepatic function, and cardiac performance. PLoS Biol, 4, e369.

Lehman, J.J., Boudina, S., Banke, N.H., Sambandam, N., Han, X., Young, D.M., Leone, T.C. et al. 2008. The transcriptional coactivator PGC1alpha is essential for maximal and efficient cardiac mitochondrial fatty acid oxidation and lipid homeostasis. Am. J. Physiol. Heart Circ. Physiol., 295, H185-H196.

Marin-Garcia, J., Goldenthal, M.J., Moe, G.W. 2001. Abnormal cardiac and skeletal muscle mitochondrial function in pacing-induced cardiac failure. Cardiovasc. Res., 52, 103-110.

Mettauer, B., Zoll, J,. Garnier, A., VenturaClapier, R. 2006. Heart failure: a model of cardiac and skeletal muscle energetic failure. Pflugers Arch., 452, 653-666.

McFarland, R., Turnbull, D.M. 2009. Batteries not included: diagnosis and management of mitochondrial disease. J. Inter. Med., 265: 210-28.

Nunnari, J., Suomalainen, A. 2012. Mitochondria: in sickness and in health. Cell, 148: 1145-59.

Okuno, D., Iino, R., Noji, H. 2011. Rotation and structure of FoF1-ATP synthase. $J$ Biochem, 149: 655-64.

Puigserver, P,. Spiegelman, B.M. 2003. 
Peroxisome proliferator-activated receptor-gamma coactivator 1 alpha (PGC-1 alpha): transcriptional coactivator and metabolic regulator. Endocr. Rev., 24, 78-90.

Sack, M.N., Kelly, D.P. 1998. The energy substrate switch during development of heart failure: gene regulatory mechanisms (Review). Int. J. Mol. Med,. 1, 17-24.

Sharov, V.G., Goussev, A., Lesch, M., Goldstein, S., Sabbah, H.N. 1998. Abnormal mitochondrial function in myocardium of dogs with chronic heart failure. J. Mol. Cell Cardiol., 30, 1757-1762.

Sharov, V.G., Todor, A.V., Silverman, N., Goldstein, S., Sabbah, H.N. 2000. Abnormal mitochondrial respiration in failed human myocardium. J. Mol. Cell Cardiol., 32, 2361-2367.

Sebastiani, M., Giordano, C., Nediani, C., Travaglini, C., Borchi, E., Zani, M., Feccia, M. et al. 2007. Induction of mitochondrial biogenesis is a maladaptive mechanism in mitochondrial cardiomyopathies. $J$. Am. Coll. Cardiol., 1362-1369.

Sun, C.K., Chang, L.T., Sheu, J.J., Wang, C.Y., Youssef, A.A., Wu, C.J. et al. 2007. Losartan preserves integrity of cardiac gap junctions and PGC- 1 alpha gene expression and prevents cellular apoptosis in remote area of left ventricular myocardium following acute myocardial infarction. Int. Heart $J ., 48,533-546$.

Scarpulla, R.C. 2008. Transcriptional paradigms in mammalian mitochondrial biogenesis and function.
Physiol. Rev., 88, 611-638.

Schaefer, A.M., McFarland, R., Blakely, E.L., He, L., Whittaker, R.G., Taylor, R.W,. et al. 2008. Prevalence of mitochondrial DNA disease in adults. Ann. Neurol., 63: 35-9.

Vega, R.B., Huss, J.M., Kelly, D.P. 2000. The coactivator PGC-1 cooperates with peroxisome proliferator-activated receptor alpha in transcriptional control of nuclear genes encoding mitochondrial fatty acid oxidation enzymes. Mol. Cell Biol,. 20, 18681876.

Vander Giezen, M., Tovar, J. 2005. Degenerate mitochondria. EMBORep, 6, 525-30.

Ventura-Clapier, R., Garnier, A., Veksler, V. 2008. Transcriptional control of mitochondrial biogenesis: the central role of PGC-1. Cardiovasc Res., 79, 208-217.

Voelker, D.R. 2009. Genetic and biochemical analysis of non-vesicular lipid traffic. Ann. Rev. Biocem., 78: 827-56.

Wu, Z., Puigserver, P., Andersson, U., Zhang, C., Adelmant, G., Mootha, V., Troy, A. et al. 1999. Mechanisms controlling mitochondrial biogenesis and respiration through the thermogenic coactivator PGC-1. Cell, $98,115-124$.

Witt, H., Schubert, C., Jaekel, J., Fliegner, D., Penkalla, A., Tiemann, K., Stypmann, J. et al. 2008. Sex-specific pathways in early cardiac response to pressure overload in mice. J. Mol. Med., 86: 1013-1024.

\section{How to cite this article:}

Swati Sharma and Abha Swarup. 2016. Mitochondrial Biogenesis in Heart: Way of Nurturing and Failure. Int.J.Curr.Microbiol.App.Sci. 5(7): 474-481. doi: http://dx.doi.org/10.20546/ijcmas.2016.507.052 